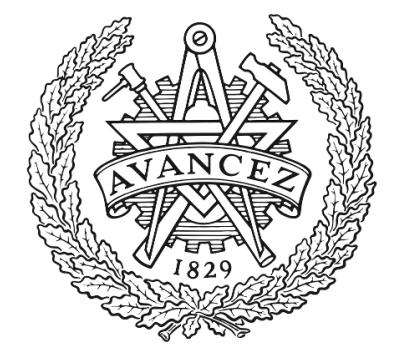

\title{
CHALMERS
}

UNIVERSITY OF TECHNOLOGY

\section{Imaging Ultrafast Dynamical Diffraction Wave Fronts in Strained Si with Coherent X Rays}

Downloaded from: https://research.chalmers.se, 2023-04-26 08:31 UTC

Citation for the original published paper (version of record):

Rodriguez-Fernandez, A., Diaz, A., Harihara Subramonia Iyer, A. et al (2021). Imaging Ultrafast Dynamical Diffraction Wave Fronts in Strained Si with Coherent X Rays. Physical Review Letters, 127(15). http://dx.doi.org/10.1103/PhysRevLett.127.157402

N.B. When citing this work, cite the original published paper. 


\title{
Imaging Ultrafast Dynamical Diffraction Wave Fronts in Strained Si with Coherent X Rays
}

\author{
Angel Rodriguez-Fernandez $\odot$, ${ }^{1,}$ Ana Diaz $\odot,{ }^{2}$ Anand H. S. Iyer, ${ }^{3}$ Mariana Verezhak, ${ }^{2}$ Klaus Wakonig, ${ }^{2}$ \\ Magnus H. Colliander $\oplus^{3},{ }^{3}$ and Dina Carbone $\oplus^{4}$ \\ ${ }^{1}$ European X-Ray Free Electron Laser GmbH, Schenefeld DE-22869, Germany \\ ${ }^{2}$ Paul Scherrer Institute, Forschungsstrasse 111, Villigen PSI, Switzerland CH-5232 \\ ${ }^{3}$ Department of Physics, Chalmers University of Technology, Gothenburg, Sweden SE-41296 \\ ${ }^{4}$ MAX IV Laboratory, Lund University, Lund, Sweden SE-22100
}

(Received 17 December 2020; revised 23 June 2021; accepted 26 August 2021; published 8 October 2021)

\begin{abstract}
Dynamical diffraction effects in thin single crystals produce highly monochromatic parallel x-ray beams with a mutual separation of a few microns and a time delay of a few femtoseconds - the so-called echoes. This ultrafast diffraction effect is used at X-Ray Free Electron Lasers in self-seeding schemes to improve beam monochromaticity. Here, we present a coherent x-ray imaging measurement of echoes from $\mathrm{Si}$ crystals and demonstrate that a small surface strain can be used to tune their temporal delay. These results represent a first step toward the ambitious goal of strain tailoring new x-ray optics and, conversely, open up the possibility of using ultrafast dynamical diffraction effects to study strain in materials.
\end{abstract}

DOI: 10.1103/PhysRevLett.127.157402

The emergence of fourth generation synchrotron sources based on low-emittance rings [1] and of spatially coherent $\mathrm{X}$-ray free electron lasers (XFELs) [2,3] open a new era in the study of high-coherence materials, such as perfect crystals, using high-coherence probes [4]. Recent years have seen an increase in the development and use of inverse microscopy techniques based on coherent $x$ rays [5-11] and other types of speckle analysis [12,13] for the study of crystalline materials, as well as for the characterization of coherent X-ray beams [5,14-17]. These methods are based on the assumption of a Fourier transform relation between the sample's electron density and the x-ray scattered field, derived from the so-called kinematical approximation [18]. On the other end, large single crystals are one of the primary optical elements for x-ray experiments. For these, more complex interactions including multiple diffraction, absorption, and refraction effects must be considered, which are well described by the dynamical diffraction theory [18-20]. Dynamical diffraction effects have already proven very useful for applications in ultrafast x-ray optics [21]. Nevertheless, dynamical diffraction can arise already from samples of modest dimensions $(\sim 500 \mathrm{~nm})$ and, in conjunction with the use of coherent nanobeams, have already been shown to be not only measurable $[22,23]$ but also a hindrance to standard inversion algorithms for digital microscopies [24] and requiring adaptive solutions [25].

Here, we show that the exceptional coherence properties of the x-ray beam produced at MAX IV Laboratory can be exploited to understand dynamical diffraction effects in crystalline samples and how the wave front changes in the presence of strain and propose an approach to analyze them. The effect under study, presented theoretically in
Ref. [26], consists of the appearance of multiple monochromatic beams in the diffracted and forward directions, which present a transverse displacement of a few microns between each other. These beams, also known as echoes, are delayed between each other by few femtoseconds depending of the thickness of the crystal and energy of the X-ray beam. This ultrafast effect is used in x-ray optics at XFELs for self-seeding radiation production [21]. We have previously demonstrated the transverse displacement of the echoes in diamond plates [27] and Si wafers [28] using a $2 \mu \mathrm{m}$ beam focused onto an optically coupled $\mathrm{x}$-ray detector with submicron resolution. In this Letter, we improve the resolution of our previous works and demonstrate that localized surface strain in a Si wafer can produce a spatial modulation of the echoes that translates into the tuning of their time delay.

A nanobeam is used to select regions of a $\mathrm{Si}$ sample where a residual strain field was created by nanoindentation. The coherence of the $\mathrm{x}$ rays is exploited to measure the finely structured echoes, strongly dependent on the local strain field, with a sub-100 nm resolution only achievable with inverse microscopy. The technique used, known as $\mathrm{x}$-ray teleptychography [29], is capable of measuring the $\mathrm{x}$-ray diffracted field, using as a probe a small pinhole placed after the sample [30]. In this way, no assumption is made on the interaction of $\mathrm{x}$ rays with the sample and this approach can be extended to study dynamical diffraction effects. A similar configuration, using a large coherent beam, with the sample in the diffraction condition for strain sensitivity, has been recently shown in $[31,32]$. The results presented are corroborated by simulations based on dynamical diffraction theory. This Letter provides a very 
fine tool to understand the role of dynamical diffraction in the study of ultrafast processes in perfect and strained thin crystals. With this knowledge, new x-ray optics for highcoherence sources can be designed when a better control of the temporal dependence of the diffracted signal is needed. Finally, this Letter proposes an approach for the study of strained microcrystals and time-resolved lattice deformation [33] in highly ordered materials when dynamical effects are dominant, using the echoes as a probe.

The samples used in this Letter are two $\mathrm{Si}(100)$ wafers of $100 \mu \mathrm{m}$ thickness. On the surface of one of them, a series of nanoindents are performed using an Alemnis nanoindenter inside a Zeiss Leo Ultra 55 field emission gun scanning electron microscope, as shown in Fig. S1 of the Supplemental Material [34]. Each indent produces a strain field that propagates radially from the indent with an amplitude that decreases exponentially with distance $[35,36]$. The Si samples are measured with $\mathrm{x}$ rays using a Laue geometry, with the $\mathrm{Si}(111)$ planes in diffraction conditions, so that the forward diffraction (FD) signal can be measured, as described in our previous work [27,28]. A schematic of the sample geometry is shown in Fig. 1. The FD signal can be only described using dynamical diffraction theory $[18,19,26]$, which includes all x-ray interactions within the crystal unit, beyond the diffraction of the incident beam with the directly illuminated volume. Because of the crystal's long-range order, the diffracted photons see more lattice planes also in the diffraction condition. Therefore, the photons can be diffracted multiple times both in the diffracted and forward directions. The diffraction inside the crystal occurs in the volume enclosed by the beam size in the $y$ direction and the gray triangle in Fig. 1 in the $x-z$ plane. At the crystal exit surface, all

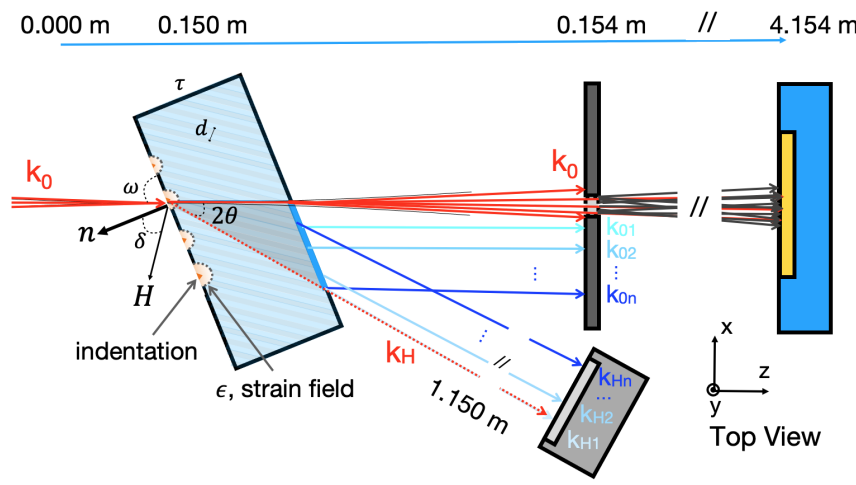

FIG. 1. Schematics of the diffraction setup. The focused x-ray beam with wavelength $\lambda$ and wave vector $\mathbf{k}_{\mathbf{0}},\left|\mathbf{k}_{\mathbf{0}}\right|=2 \pi / \lambda$, impinges on the $\mathrm{Si}$ sample oriented with a family of atomic planes (with spacing $d$ ) in diffraction conditions, $\mathbf{k}_{\mathbf{0}}+\mathbf{H}=\mathbf{k}_{\mathbf{H}}$, with $|\mathbf{H}|=2 \pi / d$. The photons are diffracted at an angle $2 \theta$ along $\mathbf{k}_{H}$. Echoes in the forward (diffraction) direction are indicated with their wave vectors $\mathbf{k}_{\mathbf{0 i}}\left(\mathbf{k}_{\mathbf{H i}}\right)$. The position of the indents on the sample, the pinhole used to scan the wave front, and the two pixel detectors, one in the forward and one in the diffraction direction, are shown. diffracted waves interfere, generating the transverse displaced echoes [26,27], both in the forward and diffracted directions, with wave vectors $\mathbf{k}_{\mathbf{0 i}}$ and $\mathbf{k}_{\mathbf{H i}}$, respectively, with $i=1,2, \ldots$ the number of the $\mathrm{x}$-ray beams generated. Because of the different paths the waves follow inside the crystal, they accumulate a temporal delay with respect to each other [26,27]. The time delay $\Delta t$ and the transverse displacement $\Delta x$ associated with each beam are related linearly [26] by

$$
\Delta x=c \cot (\theta) \Delta t,
$$

where $c$ is the speed of light and $\theta$ is the Bragg angle [37].

The measurements are performed at the beam line NanoMAX using a photon energy of $8 \mathrm{keV}$ $(\lambda=0.155 \mathrm{~nm})$ selected by a $\mathrm{Si}(111)$ monochromator. At this energy, the flux at the sample is of $10^{11} \mathrm{ph} / \mathrm{s}$ and the Kirkpatrick-Baez (KB) mirrors produce a highly coherent $\mathrm{x}$-ray focused beam with $\sim 110 \mathrm{~nm}$ waist and $250 \mu \mathrm{m}$ focal depth, with a divergence of $\sim 1.2 \mathrm{mrad}$ in both horizontal and vertical directions [17]. The Si samples are mounted on a scanning stage in the KB focal plane. A $3 \mu \mathrm{m}$ diameter pinhole is mounted on a second piezo scanning stage $3.68 \mathrm{~mm}$ downstream the sample along the beam propagation axis, as shown schematically in Fig. 1. The asymmetric $\mathrm{Si}(111)$ reflection, $\theta_{111}=14.3^{\circ}$, is chosen to match the $\sim 1 \mathrm{eV}$ monochromator bandwidth. For Laue symmetric geometry the extinction length of $\mathrm{Si}(111)$ at $8 \mathrm{keV}$ is $18.54 \mu \mathrm{m}$ and the absorption depth is $220.38 \mu \mathrm{m}$. The detector, a photon counting Merlin system with a $512 \times 512$ array of pixels with $55 \mu \mathrm{m}$ edge size, is placed $4.5 \mathrm{~m}$ downstream of the sample, to satisfy the ptychographic sampling requirement from the pinhole at the energy used. A He-filled flight tube is used to minimize air scattering between pinhole and Merlin detector. A Pilatus $100 \mathrm{~K}$ detector is placed in the horizontal scattering plane to optimize the $\mathrm{Si}(111)$ reflection.

The intensity of the forward wave front is measured with teleptychography scans [29], for both samples and both in and out of the diffraction condition. Teleptychography measurements are performed by scanning the pinhole in the $x-y$ plane, over an area of $18 \times 36 \mu \mathrm{m}^{2}$ for the strainfree sample and $18 \times 27 \mu \mathrm{m}^{2}$ for the nanoindented sample, with a step size of $0.5 \mu \mathrm{m}$. In order to counteract possible drifts of the setup, the dataset is acquired over eight separated scans, each covering an area of $10 \times 10 \mu \mathrm{m}^{2}$, with $1 \mu \mathrm{m}$ overlap between adjacent areas, as shown in Fig. S3 of the Supplemental Material [34]. Data reconstruction is performed with a tailored routine [38] that combines 500 iterations of the difference map algorithm [39] without updating the reconstruction of the pinhole for the first 499 iterations and 1500 iterations of a maximum likelihood refinement [40] with update of both wave front and pinhole. This routine applies a common refinement of the wave front in the overlapping areas, while 
the refinement of the pinhole is independent for the eight scans [41]. In the reconstruction, we use the pinhole obtained with a test specimen as an initial guess, see Fig. S4 of the Supplemental Material [34].

The teleptychography reconstructions return the amplitude and phase of the forward diffracted wave field and the transmitted beam at the pinhole position (cf. Fig. S5 of the Supplemental Material [34]). Figures 2(a) and 2(a') show the forward diffracted intensity (the square of the retrieved amplitude) from the strain-free sample out and in diffraction conditions, respectively. Echoes are indeed only seen in diffraction. These measurements reproduce previous results obtained with direct measurements [27], validating our approach. The divergence of the focused beam is not propagated through the echoes in the diffraction plane, but is only visible in the direction perpendicular to it. In the horizontal plane, indeed, the crystal acts as an x-ray beam filter diffracting only the photons with a divergence comparable with the Darwin width of the selected diffraction peak, which in our case is of $100 \mathrm{nrad}$ [18]. In the vertical plane, this filter effect does not apply [cf. Fig. 2(a')]. Nevertheless, the transmitted beam preserves the full divergence of the incoming beam, overlapping with the echoes at large distance from the sample. Figures 2(b) and 2( $\left.b^{\prime}\right)$ show the diffracted intensities of Figs. 2(a) and 2(a') at the sample plane, obtained by the propagation of the retrieved wave field $[29,32]$. The sample plane coincides with the location of the x-ray beam focus and, therefore, provides the best possible resolution of the forward diffracted field. Here, we note that there is no strict correspondence, in real space, between the position of the sample in the focus of the beam, its thickness, unintentionally
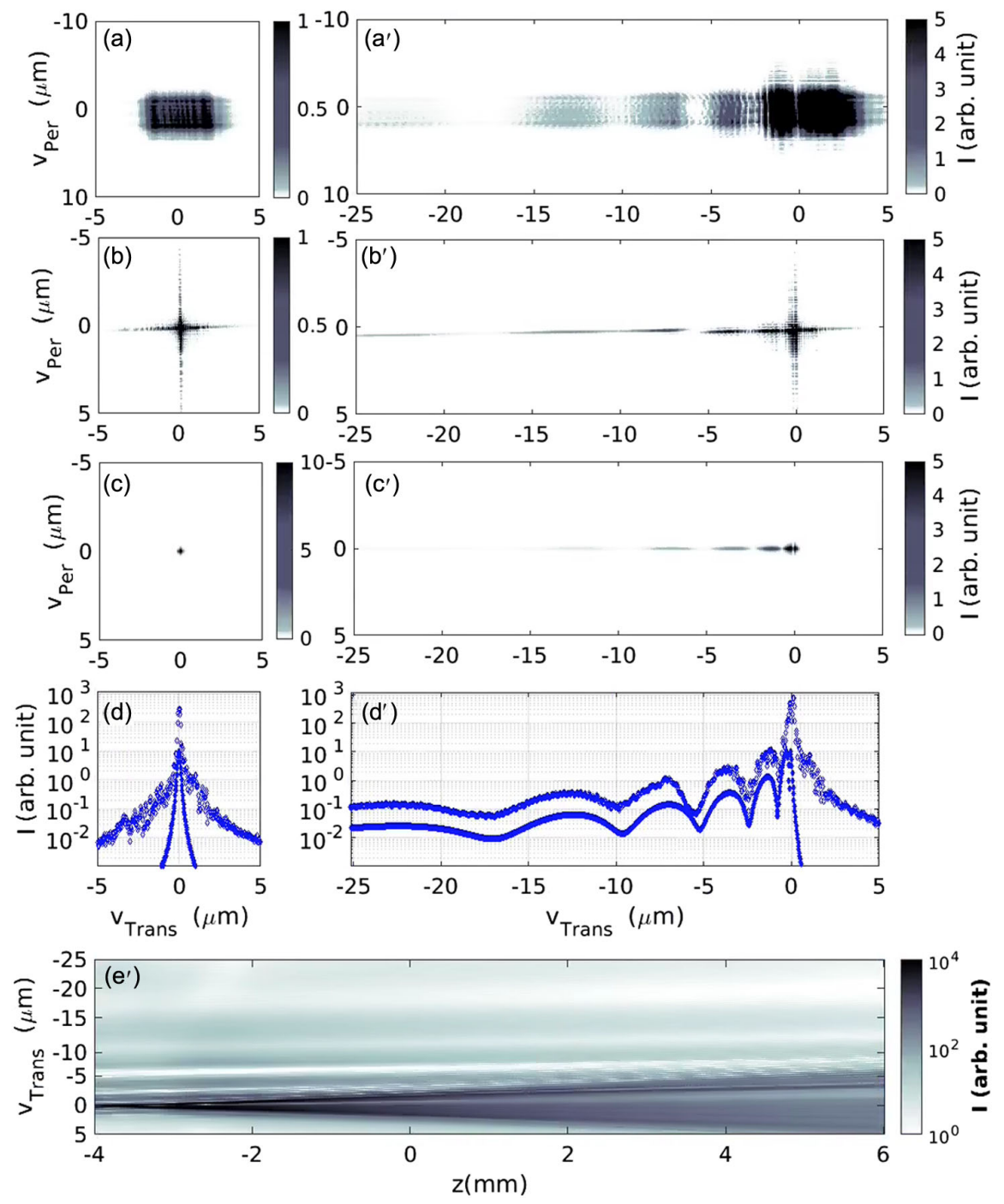

FIG. 2. Intensity of the FD wave front from strain-free Si wafer at two angles: (left) $1^{\circ}$ away-echoes are absent-and (right) at the diffraction angle. (a), (a') Reconstruction at the pinhole plane. (b), (b') Reconstruction propagated to the KB focus. (c), (c') Simulation at the focus. (d), (d') Line cuts through (a),(a') and (b), ( $\left.b^{\prime}\right)$. The simulated signal, lower line, is offset for clarity. (e') Propagation of ( $\left.a^{\prime}\right)$ along the beam direction $z$ up to the sample position, illustrating the divergence of the transmitted beam and the parallel echoes. 
comparable to the beam focal depth, and the propagation of the echoes in the focal plane. Indeed, the diffracted field measured is produced at the sample exit surface, and the backpropagation of the wave field beyond this point does not reflect any physical reality. The pixel size obtained for the reconstruction is $32 \mathrm{~nm}$, while the resolution is estimated to be $55 \mathrm{~nm}$, using Fourier ring correlation [42] for two different scans of the forward beam out of diffraction conditions (see Fig. S6 in the Supplemental Material [34]). Figures 2(c) and 2(c') show the simulation of the intensity of the forward diffracted field at the sample position for the configuration used, i.e., a $100 \mu \mathrm{m}$ thick Si crystal at the asymmetric (111) reflection, using a horizontal diffraction geometry and a strain-free model, as described in [27]. The agreement with simulations is more clearly seen in the line cuts shown in Figs. 2(d) and 2(d'), where the data are offset for clarity. Figure $2\left(\mathrm{e}^{\prime}\right)$ shows a map of the intensity of the echoes along the beam propagation direction. Because of the beam divergence, already at a distance of $2 \mathrm{~mm}$ from the sample surface, the echoes are overlapping with the divergent transmitted beam. Moreover, the echoes are parallel to the beam propagation direction, as expected.

From the indented sample, data are collected at different positions to compare the FD wave fronts produced by different strain fields. The dynamical diffraction goes far beyond the volume affected by the indent-induced strain. As a consequence, the echoes are still produced with a large contribution from the strain-free part of the Si crystal. Simulations of the expected echoes distribution are performed with our code $[27,28]$ that we have adapted to model strained crystals following the work in Refs. [43-45]. The model assumes that the indent produces an isotropic strain field of amplitude proportional to the load, which decreases with distance from the indent following an inverse exponential law. Numerically this is achieved by slicing the crystal in $20 \mathrm{~nm}$ layers parallel to the surface, each with a different value of lattice parameter following the exponential decay. This numerical step, necessary to reduce the complexity of the calculations, approximates the radial to a linear decay. Despite the fact that the approximation introduces a variation of a factor between 1 and 2 of the effective decay length $\mathrm{x}$ rays experience along all possible paths defined by the dynamical diffraction process, it still provides good correspondence between data and simulations. We expect that improving the model to reflect a true radial decay will increase this correspondence. Finally, our model also disregards the strain anisotropy expected along different crystallographic directions, due to different elastic constants. This should be included in a future model.

Figures 3(a)-3(c) show the results from the inversion of the data collected, respectively, at $1 \mathrm{~mm}$ from the indented area and close to two nanoindents with loads 25 and $75 \mathrm{mN}$. The FD signal retrieved shows an increase of the number of echoes and a decrease of their mutual distance

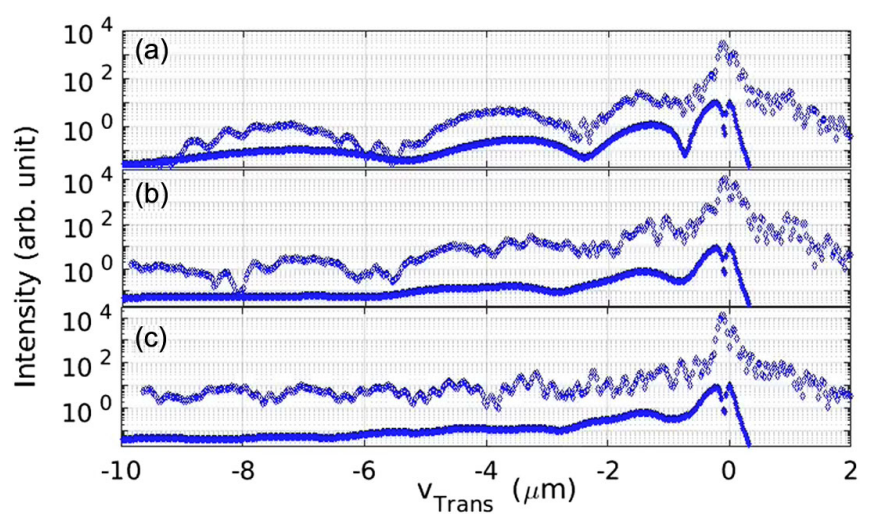

FIG. 3. Line cuts through the retrieved (empty dots) and simulated (filled dots) echoes from the indented Si sample in regions with different strain state, cf. text for details. Intensities are offset for clarity.

for increasing indentation strength. At higher strain, the echoes tend to merge into a continuum. Simulations of the dynamical diffraction signal using models with different strain fields provide a rather good agreement with data. Teleptychography reconstructions from this sample and the respective simulations are shown in Figs. S7-S10 of the Supplemental Material [34]. More accurate fitting routines will be implemented in the future. However, the position of the echoes are easily extracted via algorithms to find local maxima, as shown in Fig. S11. New echoes with shorter time delays arise in the strained areas, confirming this experimental approach to observe the strain-tailored FD maxima involved in this ultrafast process. Their time delay, visualized in Fig. S12 of the Supplemental Material, can be calculated using Eq. (1) and is shown in Fig. 4. The strain amplitude (expressed as a fractional variation of the lattice parameter) and the decay length of the exponential that best reproduces the data for the three regions (see Fig. S9 in Supplemental Material at [34]) are (i) $5 \times 10^{-5}$ over $2 \mu \mathrm{m}$, Fig. 4(a), (ii) $10^{-4}$ over $7 \mu \mathrm{m}$, Fig. 4(b), and (iii) $10^{-4}$ over

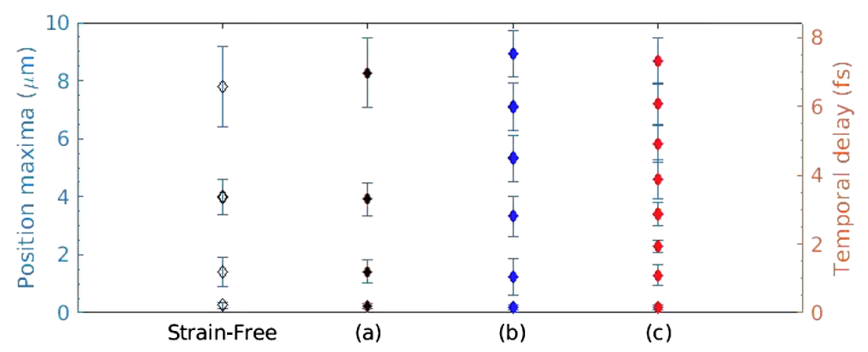

FIG. 4. (Left) Transverse displacement of the echoes along the first $8 \mu \mathrm{m}$ of the reconstruction with respect to the transmitted beam as extracted from Fig. S11 in the Supplemental Material at [34] and (right) calculated delays from Eq. (1). For the strainfree sample and the indented sample at the positions (a), (b), and (c) as presented in Fig. 3. The bars correspond to the half width at half maximum of the respective echoes. 
$10 \mu \mathrm{m}$, Fig. 4(c). These findings are in agreement with results from literature [46].

These pioneering results demonstrate the extreme sensitivity of dynamical diffraction effects to localized strain fields of small amplitude. Echoes are produced from a volume extending throughout the full sample thickness, of which the strained area represents a mere $10 \%$. Further improvement of the model will include the implementation of a radial decay of the strain field and eventually the crystal elastic anisotropy. A natural progression of this research is measuring echoes in the diffraction direction, where the spatiotemporal signal does not suffer from the absorption effects, as in FD. As presented in Fig. S13 in the Supplemental Material [34], the echoes probe the crystal in a more homogeneous manner, which allows one to retrieve more clearly the information from lattice deformations.

We have presented an experimental study of the dynamical diffraction produced by a $\mathrm{Si}$ wafer in transmission geometry, in the presence of a localized strain. We have shown that strain can be used to finely tune time delay of the echoes, in a way we are able to model. We achieve a high resolution in both sample and detection space with the use of a focused beam combined with a teleptychography approach. With the use of a nanobeam, we effectively increase the sensitivity of our measurement to strain gradients and sample heterogeneity by reducing the illuminated volume. Our approach provides a sensitive and efficient way to analyze the impact of strain of different amplitudes onto dynamical diffraction effects from perfect crystals. Conversely, understanding how dynamical diffraction is affected by the presence of strain in otherwise perfectly ordered crystals offers a new and visibly more sensitive tool for the characterization of strain and defects in crystalline materials. This Letter provides insights on the dynamical effects arising in coherent imaging experiments involving high-atomic-number crystals, such as $\mathrm{Ni}, \mathrm{Au}$, $\mathrm{InSb}$, or $\mathrm{CdTe}$, for which the extinction length reduces to the micron and submicron level, and contributes to the landscape of emerging experimental and analysis approaches developed to model them. It is important to mention that the presence from the echoes in the diffracted signal could be distorting the results in temporal ultrafast studies.

Finally, being able to predict and control the behavior of dynamical diffraction in the presence of strain opens the fascinating possibility of strain-tailoring ultrafast optics. This might include the generation of multibunch sources from single ultrafast pulses or the production of tailored crystals for split-and-delay lines with full control of the timing and width of the generated pulses with respect to the incoming pulse.

We acknowledge MAX IV Laboratory for beamtime under Proposal No. 20180253. MAX IV. is supported by the Swedish Research Council, Vinnova, and Formas under Contracts No. 2018-07152, No. 2018-04969, and
No. 2019-02496, respectively. NanoMAX staff is acknowledged for support during the preparation and the execution of the experiment. M. V. acknowledges funding by European Union's Horizon 2020 Research and Innovation Program under the Marie Skłodowska-Curie Grant Agreement No. 701647 and the SNSF Grant No. 200021L_169753. We thank Zdenek Matej, Manuel Guizar-Sicairos, Bill Pedrini, Virginie Chamard, and Kenneth Finkelstein for useful discussions. A. R.F and D. C. conceptualized the work. H. S. I. and M. H. C. prepared the samples. A.R.F., D.C., M.H.C., M. V., and A.D. planned the experiment. A.R.F., D.C., and A.D. performed the experiment. A. R. F. analyzed the data with contributions of A. D., K. W., and M. V.; A. R. F. performed the simulations. D. C. and A. R. F. wrote the Letter with the contribution of all authors.

*angel.rodriguez-fernandez@xfel.eu

[1] P. F. Tavares et al., Commissioning and first-year operational results of the MAX IV $3 \mathrm{GeV}$ ring, J. Synchrotron Radiat. 25, 1291 (2018).

[2] W. Decking et al., A MHz-repetition-rate hard x-ray freeelectron laser driven by a superconducting linear accelerator, Nat. Photonics 14, 391 (2020).

[3] J. M. Glownia et al., Time-resolved pump-probe experiments at the LCLS, Opt. Express 18, 17620 (2010).

[4] U. Pietsch, V. Holy, and T. Baumbach, High-Resolution $X$-Ray Scattering: From Thin Films to Lateral Nanostructures (Springer-Verlag, New York, 2004).

[5] P. Thibault, M. Dierolf, A. Menzel, O. Bunk, C. David, and F. Pfeiffer, High-resolution scanning x-ray diffraction microscopy, Science 321, 379 (2008).

[6] A. Ulvestad, A. Singer, H.-M. Cho, J. N. Clark, R. Harder, J. Maser, Y.S. Meng, and O. G. Shpyrko, Single particle nanomechanics in operando batteries via lensless strain mapping, Nano Lett. 14, 5123 (2014).

[7] A. Singer et al., Nucleation of dislocations and their dynamics in layered oxide cathode materials during battery charging, Nat. Energy 3, 641 (2018).

[8] P. Godard, G. Carbone, M. Allain, F. Mastropietro, G. Chen, L. Capello, A. Diaz, T. H. Metzger, J. Stangl, and V. Chamard, Three-dimensional high-resolution quantitative microscopy of extended crystals, Nat. Commun. 2, 568 (2011).

[9] M. O. Hill et al., Measuring three-dimensional strain and structural defects in a single InGaAs nanowire using coherent $\mathrm{x}$-ray multiangle Bragg projection ptychography, Nano Lett. 18, 811 (2018).

[10] S. O. Hruszkewycz, M. Allain, M. V. Holt, C. E. Murray, J. R. Holt, P. H. Fuoss, and V. Chamard, High-resolution three-dimensional structural microscopy by single-angle Bragg ptychography, Nat. Mater. 16, 244 (2017).

[11] V. Chamard, J. Stangl, G. Carbone, A. Diaz, G. Chen, C. Alfonso, C. Mocuta, and T. H. Metzger, Three-Dimensional X-Ray Fourier Transform Holography: The Bragg Case, Phys. Rev. Lett. 104, 165501 (2010). 
[12] Q. Zhang, E. M. Dufresne, and A. R. Sandy, Dynamics in hard condensed matter probed by x-ray photon correlation spectroscopy: Present and beyond, Curr. Opin. Solid State Mater. Sci. 22, 202 (2018), advanced characterization of nanomaterials.

[13] V. L. R. Jacques, C. Laulh, N. Moisan, S. Ravy, and D. Le Bolloch, Laser-Induced Charge-Density-Wave Transient Depinning in Chromium, Phys. Rev. Lett. 117, 156401 (2016).

[14] A. Schropp, P. Boye, J. M. Feldkamp, R. Hoppe, J. Patommel, D. Samberg, S. Stephan, K. Giewekemeyer, R. N. Wilke, T. Salditt, J. Gulden, A. P. Mancuso, I. A. Vartanyants, E. Weckert, S. Schder, M. Burghammer, and C. G. Schroer, Hard x-ray nanobeam characterization by coherent diffraction microscopy, Appl. Phys. Lett. 96, 091102 (2010).

[15] J. Vila-Comamala, A. Diaz, M. Guizar-Sicairos, A. Mantion, C. M. Kewish, A. Menzel, O. Bunk, and C. David, Characterization of high-resolution diffractive $\mathrm{x}$-ray optics by ptychographic coherent diffractive imaging, Opt. Express 19, 21333 (2011).

[16] A. Schropp, R. Hoppe, V. Meier, J. Patommel, F. Seiboth, H. J. Lee, B. Nagler, E. C. Galtier, B. Arnold, U. Zastrau, J. B. Hastings, D. Nilsson, F. Uhln, U. Vogt, H. M. Hertz, and C. G. Schroer, Full spatial characterization of a nanofocused $\mathrm{x}$-ray free-electron laser beam by ptychographic imaging, Sci. Rep. 3, 1633 (2013).

[17] A. Björling, S. Kalbfleisch, M. Kahnt, S. Sala, K. Parfeniukas, U. Vogt, D. Carbone, and U. Johansson, Ptychographic characterization of a coherent nanofocused x-ray beam, Opt. Express 28, 5069 (2020).

[18] W. H. Zachariasen, Theory of X-Ray Diffraction in Crystals (Dover Publications, New York, 1945).

[19] B. Batterman and H. Cole, Dynamical diffraction of x-rays by perfect crystals, Rev. Mod. Phys. 36, 681 (1964).

[20] A. Authier, Dynamical Theory of X-Ray Diffraction (Oxford University Press, New York, 2001).

[21] J. Amann et al., Demonstration of self-seeding in a hardx-ray free-electron laser, Nat. Photonics 6, 693 (2012).

[22] A. Pateras, J. Park, Y. Ahn, J. A. Tilka, M. V. Holt, H. Kim, L. J. Mawst, and P.G. Evans, Dynamical scattering in coherent hard x-ray nanobeam Bragg diffraction, Phys. Rev. B 97, 235414 (2018).

[23] M. Civita, A. Diaz, R. J. Bean, A. G. Shabalin, O. Y. Gorobtsov, I. A. Vartanyants, and I. K. Robinson, Phase modulation due to crystal diffraction by ptychographic imaging, Phys. Rev. B 97, 104101 (2018).

[24] A. G. Shabalin, O. M. Yefanov, V. L. Nosik, V. A. Bushuev, and I. A. Vartanyants, Dynamical effects in Bragg coherent x-ray diffraction imaging of finite crystals, Phys. Rev. B 96, 064111 (2017).

[25] O. Y. Gorobtsov and I. A. Vartanyants, Phase of transmitted wave in dynamical theory and quasi-kinematical approximation, Phys. Rev. B 93, 184107 (2016).

[26] Y. Shvydko and R. Lindberg, Spatiotemporal response of crystals in x-ray Bragg diffraction, Phys. Rev. ST Accel. Beams 15, 100702 (2012).

[27] A. Rodriguez-Fernandez, V. Esposito, D. F. Sanchez, K. D. Finkelstein, P. Juranic, U. Staub, D. Grolimund, S. Reiche, and B. Pedrini, Spatial displacement of forward-diffracted x-ray beams by perfect crystals, Acta Crystallogr. Sect. A 74, 75 (2018).

[28] A. Rodriguez-Fernandez et al., X-ray forward diffraction wave-front propagation in $\mathrm{Si}$ and $\mathrm{C}$ single crystals: Simulations and experiments, Proc. SPIE V 11493, 114930W (2020).

[29] E. H. R. Tsai, A. Diaz, A. Menzel, and M. Guizar-Sicairos, $\mathrm{X}$-ray ptychography using a distant analyzer, Opt. Express 24, 6441 (2016).

[30] We note that conventional ptychography, in which the sample is scanned with respect to the incoming beam, would not work in the presence of dynamical diffraction due to the assumption of a factorization of the illumination and the sample transmissivity in conventional ptychography, Ref. [5].

[31] M. Verezhak, S. Van Petegem, V. Jacques, P. Godard, K. Wakonig, L. Thilly, and A. Diaz, Visualization of crystallographic defects in InSb micropillars by ptychographic topography, Microsc. Microanal. 24, 18 (2018).

[32] M. Verezhak, S. Van Petegem, A. Rodriguez-Fernandez, P. Godard, K. Wakonig, D. Karpov, V.L. R. Jacques, A. Menzel, L. Thilly, and A. Diaz, X-ray ptychographic topography: A robust nondestructive tool for strain imaging, Phys. Rev. B 103, 144107 (2021).

[33] G. Batignani, G. Fumero, A. R. Srimath Kandada, G. Cerullo, M. Gandini, C. Ferrante, A. Petrozza, and T. Scopigno, Probing femtosecond lattice displacement upon photo-carrier generation in lead halide perovskite, Nat. Commun. 9, 1971 (2018).

[34] See Supplemental Material at http://link.aps.org/supplemental/ 10.1103/PhysRevLett.127.157402 for more information about the experimental setup and characterization of the beam and sample prior to data collection.

[35] C. Reuber, P. Eisenlohr, F. Roters, and D. Raabe, Dislocation density distribution around an indent in singlecrystalline nickel: Comparing nonlocal crystal plasticity finite-element predictions with experiments, Acta Mater. 71, 333 (2014).

[36] M. Lie, C. Lu, K. Tieu, and H. Yu, Numerical comparison between Berkovich and conical nano-indentations: Mechanical behaviour and micro-texture evolution, Mater. Sci. Eng. A 619, 57 (2014).

[37] From Bragg's law $2 d \sin \theta=n \lambda$.

[38] K. Wakonig, H.-C. Stadler, M. Odstrčil, E. H. R. Tsai, A. Diaz, M. Holler, I. Usov, J. Raabe, A. Menzel, and M. Guizar-Sicairos, Ptychoshelves, a versatile high-level framework for high-performance analysis of ptychographic data, J. Appl. Crystallogr. 53, 574 (2020).

[39] P. Thibault, M. Dierolf, O. Bunk, A. Menzel, and F. Pfeiffer, Probe retrieval in ptychographic coherent diffractive imaging, Ultramicroscopy 109, 338 (2009).

[40] P. Thibault and M. Guizar-Sicairos, Maximum-likelihood refinement for coherent diffractive imaging, New J. Phys. 14, 063004 (2012).

[41] M. Guizar-Sicairos, I. Johnson, A. Diaz, M. Holler, P. Karvinen, H.-C. Stadler, R. Dinapoli, O. Bunk, and A. Menzel, High-throughput ptychography using eiger: Scanning x-ray nano-imaging of extended regions, Opt. Express 22, 14859 (2014).

[42] M. van Heel and M. Schatz, Fourier shell correlation threshold criteria, J. Struct. Biol. 151, 250 (2005). 
[43] B. Lings, J. S. Wark, M. F. DeCamp, D. A. Reis, and S. Fahy, Simulations of time-resolved x-ray diffraction in Laue geometry, J. Phys. Condens. Matter 18, 9231 (2006).

[44] S. Takagi, Dynamical theory of diffraction applicable to crystals with any kind of small distortions, Acta. Crystallogr. 15, 1311 (1962).
[45] N. Kato and A. R. Lang, A study of Pendellösung fringes in X-ray diffraction, Acta Crystallogr. 12, 787 (1959).

[46] Z. J. Li, A. N. Danilewsky, L. Helfen, P. Mikulik, D. Haenschke, J. Wittge, D. Allen, P. McNally, and T. Baumbach, Local strain and defects in silicon wafers due to nanoindentation revealed by full-field x-ray microdiffraction imaging, J. Synchrotron Radiat. 22, 1083 (2015). 